\title{
Brief an den Herausgeber bezüglich „Mediale Schenkelhalsfraktur bei Klippel- Trenaunay-Syndrom: CT-Angiografie entscheidend für Therapie“ von C. Spink et al.
}

Mit Interesse haben wir den „interessanten Fall“ zur CT-Angiografie bei medialer Schenkelhalsfraktur bei „Klippel-Trenaunay-Syndrom“ (Referenz s. o.) gelesen. Eine pathologische Fraktur bei einem Kombinationssyndrom aus Gefäßmalformation und Weichteildysplasie stellt eine für den Patienten sehr schwerwiegende Komplikation dar und bedarf dezidierter Diagnostik und Therapie.

Nichtsdestotrotz ist hinsichtlich der verwendeten Nomenklatur, angeführten diagnostischen Schritte als auch der Schlussfolgerungen in der publizierten Kasuistik Wesentliches anzumerken.

Zunächst sollten Gefäßmalformation und assoziierte Symptome sich heutzutage an die ISSVA-Klassifikation anlehnen (Wassef M. Pediatrics 2015; 136: e203e214). „Hämangiome“ sind nicht Teil des Klippel-Trenaunay-Syndroms (KTS), wie hier zitiert. Zudem besteht beim KTS eine Skelett- und Weichteilhyperplasie, nicht eine Hypertrophie der Extremitäten. Wesentlicher Teil des Syndroms ist häufig auch eine begleitende Dysplasie (z.T. Hyperplasie, z. T. auch Hypoplasie) der Skelettmuskulatur im betroffenen Quadranten. Auch bei dem hier gezeigten Patienten ist auf der betroffenen linken Seite eine Hypoplasie des Femurs abgebildet (Abb. 1 der Kasuistik).

Zentral für die Therapie und die Differenzialdiagnose der Gefäßmalformations-/Gewebsdysplasiesyndrome ist das Vorliegen entweder einer Slow-flow-Gefäßmalformation (venös, lymphatisch, kapillär) z. B. bei einem Klippel-Trenaunay-
Syndrom, oder im Gegensatz dazu einer Fast-flow-Gefäßmalformation (arteriovenöse Malformation oder arteriovenöse Fisteln) beim Parkes-Weber-Syndrom. Die abgebildete CT-Angiografie zeigt eine Fastflow-Situation mit deutlich dilatiertem arteriellem Zustrom und frühem, dilatiertem, venösem Abstrom. Somit liegt ein Parkes-Weber-Syndrom und kein KlippelTrenaunay-Syndrom vor. Die hämodynamische Charakterisierung gelingt auch leicht durch eine farbkodierte Duplex-Sonografie/Doppler-Sonografie. Ebenso mittels einer zeitlich hoch aufgelösten, dynamischen kontrastmittelunterstützten MRAngiografie. Auch evtl. vorliegende begleitende intraossäre Gefäßmalformationen sind in der MRT sehr gut erkennbar. Insbesondere die CT-Angiografie ist hier in der Charakterisierung und Differenzialdiagnose der Gefäßmalformationen der MRT unterlegen und sollte bei den meist jungen Patienten nur in Sonderfällen angewandt werden.

Ob bei dem hier vorliegendem dysplastischem Femur auf der betroffenen linken Seite in Anwesenheit einer unbehandelten Fast-flow-Situation bei Parkes-Weber-Syndrom die konservative Therapie wirklich ausreicht, wird der Verlauf des Patienten zeigen müssen. Eine verzögerte und komplizierte Frakturheilung ist zu erwarten. Vor einer definitiven operativen Versorgung wird man hier sicherlich die Fastflow-Situation im Bereich des operativen Zugangsweges und mögliche intraossäre Fisteln interventionell durch Embolisation behandeln müssen. Insofern ist der in der
Kasuistik angesprochene interdisziplinäre Ansatz hier zwar zweifelsfrei die richtige Vorgehensweise, die Diagnose, das angewandte Verfahren und die gezogenen Schlussfolgerungen jedoch nicht adäquat.

Da die komplizierte Differenzialdiagnose und differenzierte interdisziplinäre Therapie entsprechendes Fachwissen erfordert, ist möglicherweise die Vorstellung dieser Patienten an einem dedizierten interdisziplären Zentrum für Gefäßanomalien sinnvoll.

Autoren

Walter A. Wohlgemuth ${ }^{1}$, René Mueller-Wille', Moritz Wildgruber ${ }^{2}$

1 Interdiszplinäres Zentrum für Gefäßanomalien, Institut für Röntgendiagnostik,

Universitätsklinikum Regensburg

${ }^{3}$ Institut für Klinische Radiologie,

Universitätsklinikum Münster

\section{Korrespondenzadresse}

Prof. Dr. Moritz Wildgruber Institut für Klinische Radiologie, Universitätsklinikum Münster Albert Schweitzer Campus 1 48149 Münster

Germany

moritz.wildgruber@ukmuenster.de

Bibliografie

DOI http://dx.doi.org/10.1055/s-0043-101523 Online-Publikation: 2017 | Fortschr Röntgenstr 2017; 189: 259 @ Georg Thieme Verlag KG Stuttgart · New York

ISSN 1438-9029 